\title{
The performance of lung transplantation according to the pattern of changing urgency: KONOS registry analysis
}

\author{
Jin Ho Jang ${ }^{1}$, Woo Hyun Cho ${ }^{1}$, Do Hyung Kim² ${ }^{2}$ Hye Ju Yeo ${ }^{1}$ \\ ${ }^{1}$ Division of Allergy, Pulmonary and Critical Care Medicine, Department of Internal Medicine, Pusan National University Yangsan Hospital, Yangsan, Korea \\ ${ }^{2}$ Department of Thoracic and Cardiovascular Surgery, Pusan National University Yangsan Hospital, Yangsan, Korea
}

Background: The urgency-based lung allocation system in Korea increases the proportion of lung transplantation in status 0 patients. Current system prioritizes the urgency over the waiting time. This could be disadvantageous to non-status 0 patients, which are listed early. The purpose of this study is to assess how urgency-changing pattern during waiting time on list affects lung transplantation outcome.

Methods: Based on Korean Network for Organ Sharing (KONOS) database, the results of 836 lung transplants conducted in Korea from January 2010 to December 2020 were analyzed. According to the pattern of changes of urgent status, the survival rate was compared by dividing groups into status 0 at registration (group 1), upgraded status 0 from initial status (group 2), and above status 1 (group 3).

Results: The survival rates for 1 -year and 5 -year after lung transplantation of status 0 were $68.3 \%$ and $45.9 \%$, respectively. Overall, the outcomes for status 0 were significantly lower than those for lower status $(75.8 \%, 57.8 \%$; $P<0.001)$. In each group, the 1 -year and 5 -year survival rate were $55.2 \%$ and $39 \%$ in group $1,71.9 \%$ and $52.0 \%$ in group $2,75.8 \%$ and $57.8 \%$ in group 3 , respectively. Kaplan-Meier analysis showed significantly lower survival rate in group 1 than in others (group 1 vs. 2, $P=0.01$; group 1 vs. 3 , $\mathrm{P}=0.01$; group 2 vs. $3, \mathrm{P}=0.859$ ).

Conclusions: The current KONOS status in lung transplant candidate did not discriminate the postoperative outcome. Urgency-based allocation system showed a pitfall to lead to lower survival rate in a subgroup, which were initially listed as status 0 . Refinement on allocation system in status 0 is required to improve postoperative outcome and ethical aspects, such as equality and benefit. 